\title{
Wavelet Filter Evaluation for Image Compression
}

\author{
John D. Villasenor, Benjamin Belzer, and Judy Liao
}

\begin{abstract}
Choice of filter bank in wavelet compression is a critical issue that affects image quality as well as system design. Although regularity is sometimes wed in filter evaluation, its success at predicting compression performance is only partial. A more reliable evaluation can be obtained by considering an $L$-level synthedis/analysis system as a single-input, single-output, linear shift-variant system with a response that varles according to the input location modulo $\left(2^{L}, 2^{L}\right)$. By characterizing a filter bank according to its impulse response and step response in addition to regulartty, we obtain reliable and relevant (for image coding) filter evaluation metrics. Uaing this approach, we have evaluated all possible reasomably short (less than 36 taps in the synthesis/analyds pair) minimiun-order biorthogonal wavelet filter banks. Of this group of over $\mathbf{4 0 0 0}$ candidate filter banks, we have selected and present here the filters best sulted to image compression. While some of these flters have been published previously, others are new and have properties that make them attractive in system design.
\end{abstract}

\section{INTRODUCTION}

W AVELET transforms have received significant attention recently due to their suitability for a number of important signal and image processing tasks including image coding. The principle behind the wavelet transform, as elaborated in a number of recent papers [1]-[4] is to hierarchically decompose an input signal into a series of successively lower resolution reference signals and their associated detail signals. At each level, the reference signal and detail signal (or signals in the separable multidimensional case) contain the information needed to reconstruct the reference signal at the next higher resolution level. Efficient image coding is enabled by allocating bandwidth according to the relative importance of information in the reference and detail signals and then applying scalar or vector quantization to the transformed data values [5], [6].

Many issues relating to the choice of filter bank for image compression remain unresolved. Regularity [7] has been suggested as a criterion for filter evaluation, but as a recent author noted in reference to wavelet transforms, "the importance of regularity for signal processing applications is still an open question" [8]. Furthermore, there is only a partial correlation between filter regularity and reconstructed image quality. Wavelet choice has been considered by other authors [9], [10], but the methods of these papers rest on the assumption of

Manuscript received March 6, 1994; revised October 19, 1994. This work was supported by the U.S. Department of Justice/Federal Bureau of Investigation, ARPA/CSTO under Contract J-FBI-93-112, Computer Aided Design of High Performance Wireless Networked Systems, and by Texas Instruments and Hughes Aircraft Company. The associate editor coordinating the review of this paper and approving it for publication was Prof. William A. Pearlman.

The authors are with the Electrical Engineering Department, University of California, Los Angeles, Los Angeles, CA $90024-1594$ USA.

IEEE Log Number 9412457. orthogonality, which can be incompatible with other desirable filter characteristics. The techniques we present make no assumptions regarding filter orthogonality; we are concerned instead with the relationship between data in the input signal, their representation in the reference signal, and the reconstruction to which it leads. Our work was primarily motivated by the desire to clearly characterize the relationship between the filters used in a wavelet transform and the quality of the output image in a compression scheme. We were especially interested in biorthogonal filters because they constitute an important subclass of regular, perfect reconstruction FIR filters that permit the analysis and synthesis filters to be linear phase.

Wavelet filter banks are characterized in terms of their associated continuous scaling functions and wavelets derived under iteration. This viewpoint is crucial and indeed constitutes a basis for the work presented here. However, since a biorthogonal filter bank has associated with it four such functions (the scaling functions and wavelets for the analysis and synthesis stages), it is difficult to intuitively relate the reconstructed image quality to the form of these functions. In the past, conclusions regarding the suitability of various filter banks for image coding have been made on a somewhat ad-hoc basis by compressing a set of images using several different filters. The reconstructed images with the best subjective and NMSE quality are then identified, and the properties of the scaling functions of the filter banks furnishing the best reconstruction are observed. By contrast, we adopt an end-to-end approach to the wavelet decomposition/reconstruction based on the lowest level reference signal. This allows a more intuitive inputoutput description in terms of a shift-variant linear system to be elaborated upon and clearly explains observations by previous authors regarding the greater importance of the synthesis (versus analysis) scaling function in compression performance. Our approach still requires that compression be performed experimentally on a series of impulse and step functions, but it furnishes a unified framework involving classical criteria such as impulse response, sidelobe strength, and shift-variance minimization to the characterization, evaluation, and comparison of candidate filter banks. Although one previous author [11] has proposed evaluating filter banks based on reconstruction from the reference signal, the derivation we present, allowing impulse response description in terms of the scaling functions, is new, as are the results that follow from this fundamental equation (see (14)).

Section II very briefly reviews wavelet concepts that are important to our presentation and introduces relevant notation. Section III describes our approach in which only the reference signal at the lowest resolution level is retained, allowing the entire analysis/synthesis system to be viewed as a linear, 
shift-variant system, as discussed above. We show that the impulse responses of such a system satisfy difference equations involving both the analysis and synthesis scaling functions. In Section IV, we present results and discussion and include a table giving some of the best biorthogonal filters for image compression applications.

\section{SCAling Functions, WaVElets, AND THEIR RELATION TO FILTER BANKS}

\section{A. Discrete Wavelet Transforms}

One-dimensional discrete wavelet transforms (the separable 2-D case is a straightforward extension) can be described in terms of a filter bank as shown in Fig. 1(a). An input signal $x(n)$ is input to the analysis low pass filter $h_{0}(n)$ and the analysis high-pass filter $h_{1}(n)$. The odd samples of the outputs of these filters are then discarded, corresponding to decimation by a factor of two. The decimated outputs of these filters constitute the reference signal $r_{1}(n)$ and detail signal $d_{1}(n)$ for a one-level decomposition. For reconstruction, interpolation by a factor of two is performed, followed by filtering using the lowpass and highpass synthesis filters $g_{0}(n)$ and $g_{1}(n)$ as shown. Provided that the system satisfies the perfect reconstruction property, the sum of the outputs of synthesis filters will give $y(n)=A x\left(n-n_{d}\right)$, where $A$ is a gain factor and $n_{d}$ is an odd delay.

For a multilevel decomposition, the reference signal $r_{1}(n)$ serves as the input to a filter bank whose analysis stage is identical to that of Fig. 1(a). This process, which is repeated iteratively as shown in Fig. 1(b), provides, after $L$ levels, a reference signal $r_{L}(n)$ with resolution reduced by factor $2^{L}$ with respect to the original input $x(n)$, as well as the detail signals $d_{L}(n) d_{L-1}(n), \ldots, d_{1}(n)$. Each detail signal $d_{i}(n)$ contains precisely the information that, together with the reference signal $r_{i}(n)$, enables reconstruction of $r_{i-1}(n)$, which is the reference signal at the next higher resolution.

One can associate with a multilevel analysis filter bank (similar to Fig. 1(b) but with an arbitrary number of levels in the low-pass branch) a continuous scaling function and wavelet. A generally different scaling function and wavelet are associated with the synthesis stage. References [12] and [8] contain a thorough treatment of the relationship between the filter coefficients and scaling-functions; here, we include enough discussion to make the notation clear.

The equivalent filter corresponding to $L$ levels of passage through the reference signal subtree can be expressed using the Noble identities as

$$
h^{(L)}(n)=h(n) * h(n / 2) * h(n / 4) * \ldots * h\left(n / 2^{L}\right)
$$

where we follow the usual convention that $h(n / K)=0$ whenever $n$ is not a multiple of $K \cdot H^{(L)}(n)$ is called the $L$ th iteration of the filter $h(n)$ and will have length $\left(2^{L}-\right.$ 1) $(N-1)+1$. In examining the convergence of the iterated filter to a continuous function, it is convenient to denote (after [8]) a piecewise constant function $f_{h}(x)$ associated with the $i$ th iteration of $h(n)$ through

$$
f_{h}^{(i)}(x)=2^{i / 2} h^{(i)}(n) \quad n / 2^{i} \leq x<(n+1) / 2^{i} .
$$

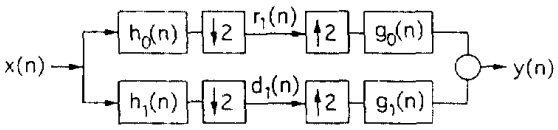

(a)

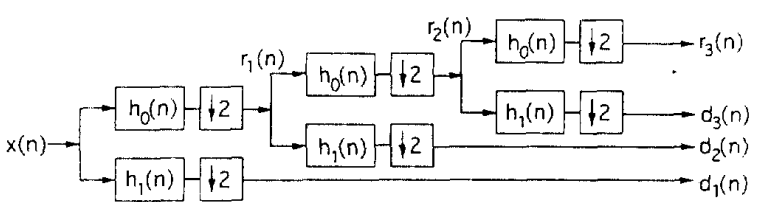

(b)

Fig. 1. (a) Basic filter bank for wavelet transformation; (b) tree structure showing three-level transform.

A filter $h(n)$ is said to be regular if $f_{h}^{(i)}(x)$ converges in the limit as $i \rightarrow \infty$ to a continuous function. In wavelet transforms, the scaling functions $\phi_{A}(x)$ and $\phi_{S}(x)$ satisfy the respective two-scale difference equations:

$$
\begin{aligned}
& \phi_{A}(x)=\sum_{n} 2^{1 / 2} h_{0}(n) \phi_{A}(2 x-n) \\
& \phi_{S}(x)=\sum_{n} 2^{1 / 2} g_{0}(n) \phi_{S}(2 x-n) .
\end{aligned}
$$

The continuous wavelets $\psi_{A}(x)$ and $\psi_{S}(x)$ are defined in terms of the scaling functions and the coefficients of the highpass, complementary filter through

$$
\begin{aligned}
& \psi_{.4}(x)=\sum_{n} h_{1}(n) \phi_{A}(2 x-n) \\
& \psi_{S}(x)=\sum_{n} g_{1}(n) \phi_{S}(2 x-n) .
\end{aligned}
$$

As will be seen below, the functions $\phi_{A}(x)$ and $\phi_{S}(x)$ are central in determining the suitability of a particular choice of filters for image compression applications.

\section{B. Filter Design}

Constraints on filter design include perfect reconstruction, finite-length, and the regularity requirement that the iterated lowpass filters involved converge to continuous functions. The regularity constraint is the crucial distinction between wavelet transforms and perfect reconstruction filter banks and can be related to the number of zeros of the $z$-transform of the lowpass filters at $z=-1$. Another important characteristic that is often sought is linear phase in all of the filters in Fig. 1(a). This precludes the use of nontrivial orthogonal filters but permits a class of filters known as biorthogonal. Biorthogonal filters differ from orthogonal filters in that the subspace spanned by the lowpass synthesis filter response, and its even shifts $g_{0}(n-2 k)$ is not orthogonal to that spanned by $g_{1}(n-2 k)$. Completeness, however, is still maintained, and any signal in $l^{2}(Z)$ has a nonzero projection in at least one of these subspaces.

Considering only the single-level system shown in Fig. 1(a), one notes that the inputs to the synthesis filters $g_{0}$ and $g_{1}$ are 
interpolated signals whose odd values are always zero. The output $y(n)$ is a linear combination of even shifts of these filter responses, and one requirement for wavelet transform filter banks is that the synthesis filter responses and their even shifts $g_{0}(n-2 k)$ and $g_{1}(n-2 k)$ form a basis spanning the space $l^{2}(Z)$. The analysis filters can be viewed as furnishing, via convolution with the input $x(n)$, the coefficients to be applied to the synthesis basis functions such that perfect reconstruction of $x(n)$ occurs at the filter bank output. From the theory of filter banks [8], one also notes that in order to eliminate aliasing, the relations

$$
\begin{aligned}
& g_{1}(n)=(-1)^{n+1} h_{0}(n) \\
& h_{1}(n)=(-1)^{n} g_{0}(n)
\end{aligned}
$$

must be satisfied and that in order to ensure perfect reconstruction, the condition

$$
p(2 n+1)=i_{n M}
$$

must hold, where $p(n)=h_{0}(n) * g_{0}(n)$, and $M$ is the index of the center tap of $p(n)$. These conditions imply a duality between the analysis and synthesis halves of the filter bank; the analysis filter banks and their even shifts will also span $l^{2}(Z)$, and reversing the roles of the analysis and synthesis filters will preserve perfect reconstruction (although the reference and detail signals will be altered by the reversal).

Design techniques for both orthogonal and biorthogonal wavelet filter banks draw heavily on the theory of perfect reconstruction filter banks. Even when all of the above constraints are applied, there remains significant flexibility in filter choice. It has been demonstrated in [8] and [12] that maximizing the number of zeros at $z=-1$ for both the analysis and synthesis filters, within reasonable constraints on the filter lengths, results in most cases in regular filters corresponding to smooth scaling functions and wavelets. It is shown in [12] that if the filter $H_{0}(z)$ is taken to be the length $2 N+1$ binomial filter $B(z)=\left(\left(1+z^{-1}\right) / 2\right)^{2 N}$, then the minimum order $G_{0}(z)$ for a perfect reconstruction system is the length $2 N-1$ remainder filter

$$
R(z)=2 \sum_{k=0}^{N-1}\left(\begin{array}{c}
N-1+k \\
k
\end{array}\right)(\dot{-1})^{k} z^{-(N-1-k)}\left(\frac{1-z^{-1}}{2}\right)^{2 k}
$$

Since $R(z)$ is linear phase, it can be factored into linear phase polynomials with real coefficients by appropriate grouping of the zeros of $R(z)$, which appear in complex quads or real reciprocal pairs (unless they happen to fall on the unit circle). Thus, for a given $N$, many different linear phase filter designs are enabled by factoring $R(z)$ into linear phase factors and allocating the factors of $B(z)$ and $R(z)$ to either $H_{0}(z)$ or $G_{0}(z)$. The number of possible linear phase filter designs based on the minimal order remainder filter $R(z)$ is $2^{N_{r}}$. where $N_{r}=(2 N-2) / 4$ if $2 N \ldots 2$ is a multiple of 4 and $N_{r}=(2 N-4) / 4+1$ otherwise. Here, we assume that $R(z)$ has no zeros on the unit circle, which is true for all reasonably short filters $(N \leq 20)$.

\section{Filter Bank Selection}

\section{A. The Wavelet Analysis/Synthesis Bank as a Linear System}

Our goal is to establish an approximate linear system model that can be used to evaluate the suitability of candidate filter banks for compression. In general, common coding techniques such as scalar and vector quantization and run-length coding preclude linear system description. However, if the number of levels $L$ is small (two or three), the performance of wavelet compression schemes is approximated by the linear system in which the information in the lowest resolution reference signal $r_{L}(n)$ is perfectly preserved, and the information in the detail signals is discarded. This is similar to actual compression algorithms, where the reference signal is coded accurately in accordance with the well-known $1 / f$ property of most natural image spectra. Since the reference signal is finely quantized while the detail signals must be coarsely quantized, it is better to use a filter bank that packs the maximum amount of information about the original image into the reference signal. In addition, the filter bank should not result in a decomposition that leads to artifacts in the image reconstructed from the reference signal alone. This is because these artifacts, which would be canceled by the information in the detail signals if lossless coding were used, can only be partially canceled when the detail signals undergo the severe compression necessary to achieve reasonable overall coding rates. It is therefore preferable to use a filter bank that leads to a relatively artifact-free reconstruction from the reference signal, with the information retained from the detail signals then used to sharpen edges and other features. This also ensures that as the coding rate is decreased and the detail signals recei ${ }^{-e}$ fewer bits or are discarded altogether, the reconstructed image will degrade gracefully and will remain relatively artifact free. By seeking a filter bank that leads to the highest fidelity reconstruction based on the reference signal, we are distributing the information in the wavelet transform domain in a manner consistent with the priority it will given in the coding.

We have found that selecting filters in this manner does in fact result in superior image quality when a full coding scheme (i.e., one in which reference and detail signals are coded) is utilized.

For a given filter bank, we can characterize the performance of a wavelet compression system in terms of a shift-variant impulse response relating an impulse $\delta\left(n-n_{i}\right)$ at the input to its reconstruction $h_{A S}^{(L)}\left(n, n_{i}\right)$ obtained using an $L$-level decomposition when only the reference signal is retained. The shift variance of the system is reflected by the parameter $n_{i}$. For a general shift-variant system with a length- $N$ input signal, there are $N$ different impulse responses as $n_{i}$ varies between 0 and $N-1$.

Since an input image and its reconstruction based on the reference signal are related by a linear shift-variant system, our approach is to identify filter banks whose associated impulse and step responses are well behaved according to classical criteria of minimum shift variance and sidelobe strength. The shift variance of wavelet transforms has been observed by Strang [4] and Mallat [3] and is viewed as one 
TABLE I

Best Biorthogonal Filter Banks for WaVelet Image Compression

\begin{tabular}{|c|c|c|c|c|c|c|c|c|}
\hline & & 薦 & $\begin{array}{l}\text { Filter coefficients (origin is leftmost coefficient, coefs for negative } \\
\text { indices follow by symmetry) }\end{array}$ & $\begin{array}{l}\text { Regu- } \\
\text { larity }\end{array}$ & $\begin{array}{l}\text { Imp. } \\
\text { Resp. }\end{array}$ & $\begin{array}{c}\text { Step } \\
\text { Resp }^{2}\end{array}$ & PSNR $^{3}$ & $\begin{array}{l}\text { Referencel } \\
\text { Comments }\end{array}$ \\
\hline \multirow[t]{2}{*}{1} & $\mathrm{H}_{0}$ & 9 & $.852699, .377402,-.110624,-.023849, .037828$ & 1.068 & \multirow[t]{2}{*}{11.224} & \multirow[t]{2}{*}{0.02707} & \multirow[t]{2}{*}{29.67} & [5] \\
\hline & $\mathrm{G}_{0}$ & 7 & $.788486, .418092,-.040689,-.064539$ & 1.701 & & & & Good overall. \\
\hline \multirow[t]{2}{*}{2} & $\mathrm{H}_{0}$ & 13 & $.767245, .383269,-.068878,-.033475, .047282, .003759,-.008473$ & 1.899 & \multirow[t]{2}{*}{10.901} & \multirow[t]{2}{*}{0.03629} & \multirow[t]{2}{*}{29.57} & \\
\hline & $\mathrm{G}_{0}$ & 11 & $.832848, .448109,-.069163,-.108737, .006292, .014182$ & 2.324 & & & & \\
\hline \multirow[t]{2}{*}{3} & $\mathrm{H}_{0}$ & 6 & $.788486, .047699,-.129078$ & 0.701 & \multirow[t]{2}{*}{11.941} & \multirow[t]{2}{*}{0.02431} & \multirow[t]{2}{*}{29.53} & \\
\hline & $\mathrm{G}_{0}$ & 10 & $.615051, .133389,-.067237, .006989, .018914$ & 2.068 & & & & shift variance. \\
\hline \multirow[t]{2}{*}{4} & $\mathrm{H}_{0}$ & 5 & $1.060660, .353553,-.176777$ & Diverg & \multirow[t]{2}{*}{10.458} & \multirow[t]{2}{*}{0.00244} & \multirow[t]{2}{*}{29.13} & \\
\hline & $\mathrm{G}_{0}$ & 3 & $.707107, .353553$ & 1.000 & & & & Integer coeffs. $^{4}$ \\
\hline \multirow[t]{2}{*}{5} & $\mathrm{H}_{0}$ & 2 & $.707107, .707107$ & 0.000 & \multirow[t]{2}{*}{17.457} & \multirow[t]{2}{*}{0.01248} & \multirow[t]{2}{*}{29.28} & \\
\hline & $\mathrm{G}_{0}$ & 6 & $.707107, .088388,-.088388$ & 1.000 & & & & Integer coeffs. ${ }^{4}$ \\
\hline \multirow[t]{2}{*}{6} & $\mathrm{H}_{0}$ & 9 & $.994369, .419845,-.176777,-.066291, .033145$ & 0.830 & \multirow[t]{2}{*}{11.080} & \multirow[t]{2}{*}{0.01523} & \multirow[t]{2}{*}{29.13} & {$[5]$} \\
\hline & $\mathrm{G}_{0}$ & 3 & $.707107, .353553$ & 1.000 & & & & Integer coeffs. ${ }^{4}$ \\
\hline \multirow[t]{2}{*}{$7^{*}$} & $\mathbf{H}_{0}$ & 2 & $.707107, .707107$ & 0.000 & \multirow[t]{2}{*}{ Inf } & \multirow[t]{2}{*}{0.00000} & \multirow[t]{2}{*}{27.48} & Haar basis. \\
\hline & $\mathrm{G}_{0}$ & 2 & $.707107, .707107$ & 0.000 & & & & Integer coeffs. ${ }^{4}$ \\
\hline \multirow[t]{2}{*}{$8 *$} & $\mathrm{H}_{0}$ & 9 & $.544089, .296844, .041409, .056710, .040100$ & 1.919 & \multirow[t]{2}{*}{10.762} & \multirow[t]{2}{*}{.04008} & \multirow[t]{2}{*}{22.66} & \multirow{2}{*}{$\begin{array}{l}\text { High reg, poor } \\
\text { performance. }\end{array}$} \\
\hline & $\mathrm{G}_{0}$ & 23 & $1.2680, .6337,-.2967,-.3712, .0072, .1098, .0116,-.0212,-.0027$, etc & 3.437 & & & & \\
\hline
\end{tabular}

1. Minimum peak to sidelobe ratio in $\mathrm{dB}$ among the eight possible impulse responses in a three-level decomposition.

2. Average fractional overshoot of second sidelobe of step response. A strong overshoot will lead to significant ringing in the reconstructed image.

3. Average PSNR in $\mathrm{dB}$ for 8 test images using a three level transform with adaptive scalar quantization of detail signals.

4. A single normalizing factor can be chosen to make all coefficients integers.

* Filters 7 and 8 give poorer performance but are included to illustrate points made in text. Filter 7 is the Haar basis and has zero regularity and good impulse and step responses. Filter 8 is a filter with high regularity but poor compression performance, and illustrates that regularity alone is not a sufficient criterion for filter choice.

of the principal disadvantages of applying wavelet techniques to signal and image processing tasks. Simoncelli et al. [14] have extended the wavelet transform shift invariance issue to include considerations of scale and, in two dimensions, orientation. They propose a more general term-shiftable multiscale transforms-to encompass these properties and derive transforms in which the information within a given detail signal remains invariant under translation or rotation. While this work addresses the important issue of conservation of information within a given subband, it does not directly treat the question of shift-variance of the combined analysis/synthesis system. Given a coding scheme in which an input signal $x(n)$ is decomposed and then reconstructed to furnish $y(n)$, we wish to minimize the sensitivity of the reconstruction to shifts of the input. While strict shift invariance is not achievable, there is an enormous variety in the degree of shift variance associated with different biorthogonal filters. A simple example will suffice to illustrate this point. Fig. 2(a) shows all of the possible (excluding symmetries) impulse responses associated with a three-level 1-D transform using filter 1 from Table I. Although the impulse response is a function of input location modulo 8 , symmetries reduce the number of distinct curves to five for odd length filters and four for even length filters. We have plotted all these responses on

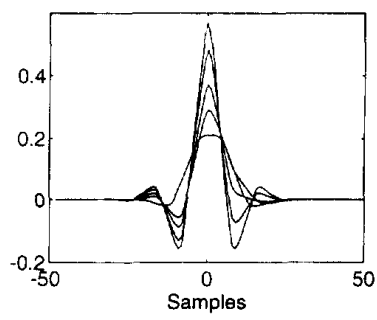

(a)

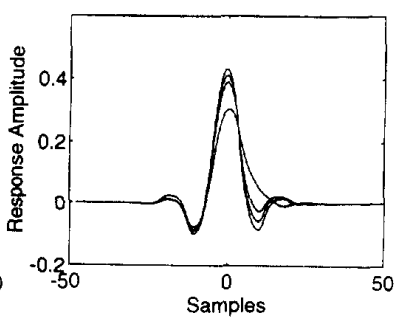

(b)
Fig. 2. Comparison of shift variance for filters (a) 1 and (b) 3 from Table I. In each case, all of the impulse responses (excluding symmetries) occurring when a three-level transform is used are shown. In a shift-invariant system, all impulse responses would be identical. The filter used in Fig. 2(b) clearly exhibits a lower degree of shift variance than the filter used in Fig. 2(a).

the same graph to emphasize the variation that occurs as the input location is changed. Fig. 2(b) shows the corresponding curves associated with filter 3 from Table I. While both filter banks display shift variance, the extent of shift variance is clearly larger in Fig. 2(a).

An additional goal is to minimize oscillatory behavior, or ringing, in the system response, as this leads to highly objectionable artifacts in the reconstructed image. To achieve this, we select filters resulting in impulse responses having a favorable peak to sidelobe ratio. In addition, we examine the 
step responses and identify filters with low "undershoots" (the second oscillation, which occurs immediately after the initial overshoot). As with shift invariance, substantial variation exists in oscillatory behavior among valid biorthogonal filter banks. A further complication arises because impulse and step responses are, of course, shift variant themselves and must be somehow combined into a single measure. Simultaneous optimization of shift variance, impulse response peak to sidelobe ratio, step response oscillations, and regularity is possible, but finding the proper weighting of these characteristics that yields superior filters for image compression is still an open question. In addition, we have found that our assumption that 1-D metrics completely characterize the performance of filters for 2-D image compression is not fully justified. There are other filter characteristics, depending on the interaction between the horizontal and vertical filter responses, that are important in determining a filter's performance in image compression. For the work discussed here, we searched the space of valid biorthogonal filter solutions for filters achieving a reasonable compromise with respect to the metrics under study.

\section{B. Impulse Response derivation}

Consider the input sequence $x(n)=\delta\left(n-n_{i}\right)$, where $n_{i}$ is located sufficiently far from the boundaries so that edge effects do not occur. After one level of decomposition, the reference signal $r_{1}(n)$ will be the result of convolving $x(n)=\delta\left(n-n_{i}\right)$ with the lowpass synthesis filter $h_{0}(n)$ followed by decimation by a factor of two. Depending on whether $n_{i}$ is even or odd, $r_{1}(n)$ will contain either the even or odd coefficients of the filter $h_{0}(n)$. The reference signal $r_{2}(n)$ is obtained by convolving $r_{1}(n)$ with $h_{0}(n)$ and decimating the result, and the general case of $L$ levels entails $L$ filter/decimate operations. The analysis impuise response $h_{A}^{(L)}\left(n, n_{i}\right)$ at the output of the final decimator is obtained by retaining one out of every $2^{L}$ samples and can be expressed as samples of the piecewise constant function $f_{h 0}^{(L)}$ associated with the $L$ th iteration of $h_{0}(n)$ :

$$
h_{A}^{(L)}\left(n, n_{i}\right)=f_{h 0}^{(L)}\left(n-\frac{n_{i}}{2^{L}}\right)
$$

Note that the analysis impulse response is finite duration because it consists of samples of a compactly supported function $f_{h 0}^{(L)}(x)$. Let the lengths of the analysis and synthesis lowpass filters be $N_{A}$ and $N_{S}$, respectively. Depending on the location $n_{i}$ of the delta function and on whether $N_{A}$ is even or odd, $h_{A}^{(L)}\left(n, n_{i}\right)$ will contain up to $N_{A}-1$ nonzero values. Furthermore, since $h_{A}^{(L)}\left(n, n_{i}\right)$ is composed of unit-interval samples of $f_{h 0}^{(L)}(x)$ offset by $n_{i} / 2^{L}$, there are, in general, $2^{L}$ different impulse responses that can occur. When symmetry is considered, the number of distinct impulse responses will reduce to $2^{L-1}$ when $N_{A}$ is even and $2^{L-1}+1$ when $N_{A}$ is odd. The particular impulse response that will be expressed for a given $n_{i}$ depends on $n_{i} \bmod 2^{L}$.

Now, consider a reference signal of the form $r_{L}(n)=$ $\delta\left(n-n_{j}\right)$, where $0 \leq n \leq N / 2^{L}$. Provided that the detail signals $d_{i}(n)$ are equal to zero for $1 \leq i \leq L$, the reference signal at level $L-1$, which is found by interpolating $r_{L}(n)$ and filtering it with $g_{0}(n)$, will be $r_{L-1}(n)=g_{0}\left(n-2 n_{j}\right)$. To generate the reference signal at level $L-2$, interpolation followed by filtering with $g_{0}(n)$ is performed again. This procedure, which is repeated a total of $L$ times, generates a shifted version of the $L$ th iteration of the filter $g_{0}(n)$. The synthesis impulse response relating the reference signal $r_{L}(n)=\delta\left(n-n_{j}\right)$ and the reconstructed sequence it generates is therefore

$$
h_{S}^{(L)}\left(n, n_{j}\right)=f_{g 0}^{(L)}\left(\frac{n}{2^{L}}-n_{j}\right)
$$

Note that in contrast with the analysis impulse response (see (8)), which has approximately constant length regardless of the number of levels $L$, the synthesis impulse response $h_{S}^{(L)}\left(n, n_{j}\right)$ has a length that grows with $L$ according to $\left(2^{L}-1\right)\left(N_{S}-1\right)+1$. In addition, because the reconstruction involves interpolation rather than decimation, the numerical values contained in the response $h_{S}^{(L)}\left(n, n_{j}\right)$ are independent to within a shift of $n_{j}$.

Combining the synthesis impulse response $h_{S}^{(L)}\left(n, n_{j}\right)$ with the analysis impulse response $h_{A}^{(L)}\left(n, n_{i}\right)$ gives the impulse response $h_{A S}^{(L)}\left(n, n_{i}\right)$ of the combined $L$-level analysis/synthesis system. Noting that reconstruction from a general reference signal is the linear combination

$$
\sum_{k} r_{L}(k) f_{g 0}^{(L)}\left(\frac{n}{2^{L}}-k\right)
$$

and that when the input is $x(n)=\delta\left(n-n_{i}\right)$, the reference signal will be

$$
r_{L}(n)=h_{A}^{(L)}\left(n, n_{i}\right)=f_{h 0}^{(L)}\left(n-\frac{n_{i}}{2^{L}}\right)
$$

Substituting (11) into (10) gives

$$
h_{A S}^{(L)}\left(n, n_{i}\right)=\sum_{k} f_{h 0}^{(L)}\left(k-\frac{n_{i}}{2^{L}}\right) f_{g 0}^{(L)}\left(\frac{n}{2^{L}}-k\right)
$$

We can associate the impulse response with a piecewise continuous function in the manner of (8)

$$
\begin{gathered}
f_{A S}^{(L)}(x, \Delta x)=2^{L / 2} h_{A S}^{(L)}\left(n, n_{i}\right) \\
n / 2^{L} \leq x<(n+1) / 2^{L}
\end{gathered}
$$

where $\Delta x=n_{i} / 2^{L}$, and expresses the offset of the input delta function as a fraction of $2^{L}$. When the number of levels $L$ becomes infinite, we have $f_{h 0}^{(\infty)}(x)=\phi_{A}(x)$ and $f_{g 0}^{(\infty)}(x)=\phi_{S}(x)$, and the impulse response converges to a family of continuous functions given by

$$
\begin{aligned}
\lim _{L \rightarrow \infty} f_{A S}^{(L)}(x, \Delta x) & =f_{A S}^{(\infty)}(x, \Delta x) \\
& =\sum_{i} \phi_{A}(i-\Delta x) \phi_{S}(x-i)
\end{aligned}
$$




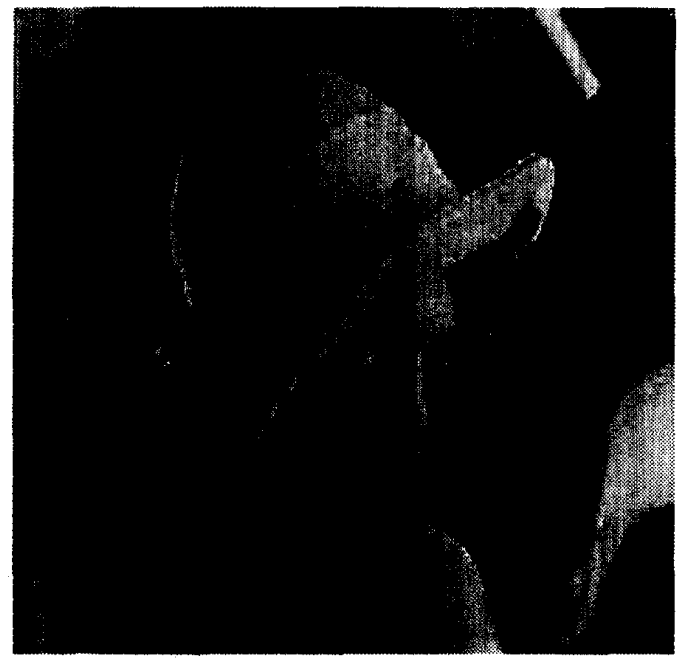

(a)

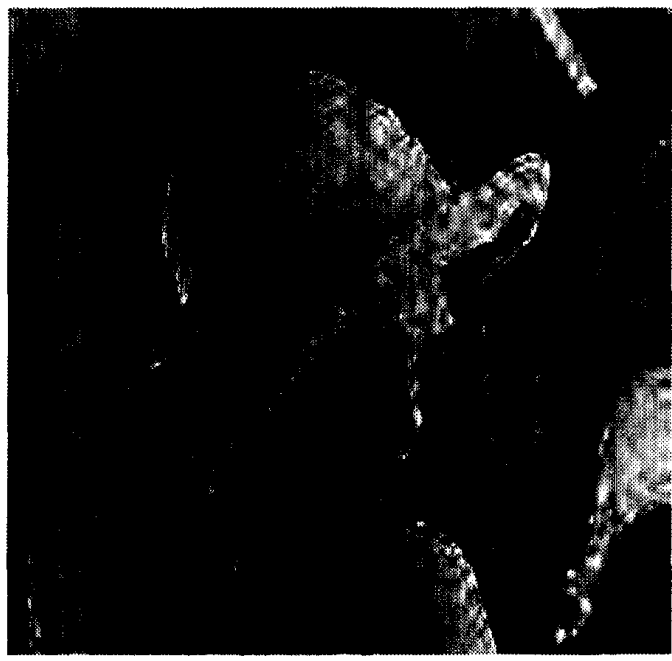

(b)

Fig. 3. Case in which filter regularity fails as a predictor of compression performance. Both $256 \times 256$ images are the result of compression at 0.5 b/pixel with a 3 level DWT followed by adaptive quantization and entropy coding. For Fig. 3(a), filter \#5 from Table I was used and attained 30.23 dB PSNR despite having low analysis/synthesis regularity of $0.00 / 1.00$. For Fig. 3(b), filter \#8 from Table I was used. Filter \#8 has relatively high analysis/synthesis regularity of $1.92 / 3.44$ yet attains only $23.19 \mathrm{~dB}$ PSNR. Part of the discrepancy in image quality can be explained by the fact that filter $\# 5$ has significantly better step and impulse response performance than filter \#8 (see Table I)

\section{RESULTS AND DISCUSSION}

Equation (12) shows that, in general, the impulse response of a wavelet filter bank is a linear combination of the iterated synthesis lowpass filter response using weights that are samples of the iterated analysis lowpass filter response. In the limit of large $L$, the impulse response becomes a combination of the synthesis scaling function and its unit shifts, with weights that are samples of the analysis scaling function. Several important practical properties of wavelet transforms follow directly from the limiting case expressed in (14).

First, the impulse response will be a linear combination of approximately $N_{A}$ shifted versions of $\phi_{S}(x)$. As a result, the smoothness of $f_{A S}^{(\infty)}(x)$ is essentially the same as that of $\phi_{S}(x)$. This explains the greater importance of the synthesis scaling function (relative to the analysis scaling function) in determining the quality of a reconstructed image. If in a given filter bank one of the scaling functions has sharp edges while the other is smooth, it is better to choose the smooth function for the synthesis filter. If the analysis filter is short, $\phi_{A}(i)$ will contain only a few nonzero values, and the undesirable properties of the analysis filter will not generally be passed on to the impulse response.

Although longer analysis filters will cause a greater number of shifted versions of $\phi_{S}(x)$ to be summed to give the impulse response, the width of the main lobe of $\phi_{A}(x)$ will be approximately constant, regardless of $N_{A}$ in accordance with the halfband lowpass nature of $h_{0}(n)$. As a result, many of the samples of $\phi_{A}(x)$ will have very small magnitudes and will not contribute significantly to the impulse response. What does change as $N_{A}$ increases is the potential for oscillatory behavior ("ringing") of $\phi_{A}(x)$, which can increase the number of significant samples used in (12) and (14).
Once the impulse and step response are obtained for a given filter bank, they can be evaluated using any number of criteria. We have found that the most common and objectionable artifacts in reconstructed images are ringing near sharply defined feature boundaries. To select filters less likely to introduce ringing, we chose, as our measures, the peak to first sidelobe ratio of the impulse response (minimized over all shifts) and the average second sidelobe of the step response.

We evaluated and ranked all of the minimum order biorthogonal filter banks with combined analysis/synthesis filter lengths of $\leq 36$ according to the impulse and step response criteria given above and calculated the Holder regularity [7] of the analysis and synthesis filters. To empirically evaluate the performance of each filter and to allow comparison with the rankings obtained using the impulse and step responses, we also performed 16:1 compression on actual images. The coding algorithm used was a three-level wavelet transform in which the transformed data were subject to adaptive scalar quantization of the detail signals followed by entropy coding using run length and Huffman coding. The reference image was quantized using $8 \mathrm{~b} / \mathrm{pixel}$. For the detail signals, the bit allocation procedure selects the quantization step size to be proportional to $1 / \log \sigma_{i}^{2}$, where $\sigma_{i}$ is the variance of the $i$ th subband. This allocation procedure has been observed to give good results experimentally when the quantizer is followed by Huffman coding of run, level pairs. More complex quantization approaches have been observed to provide slightly better coding performance [15], [16], but since we were concerned primarily with evaluating the filters, we chose a quantization scheme that can be easily and consistently applied using different filters and input images without the need for codebook training or complicated data structures. A total of 


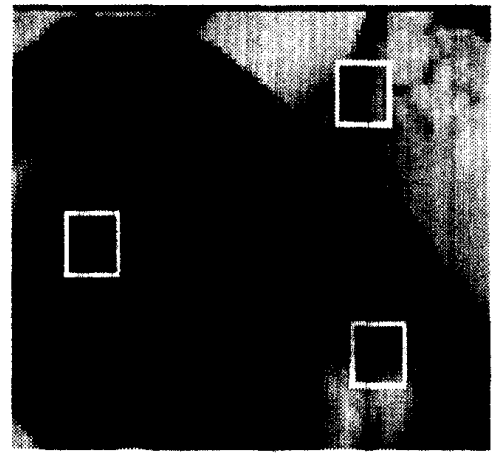

(a)

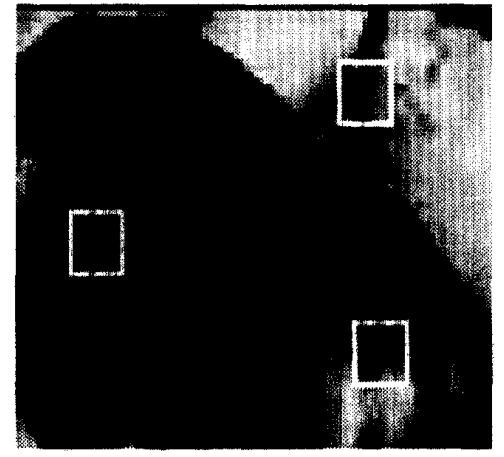

(b)

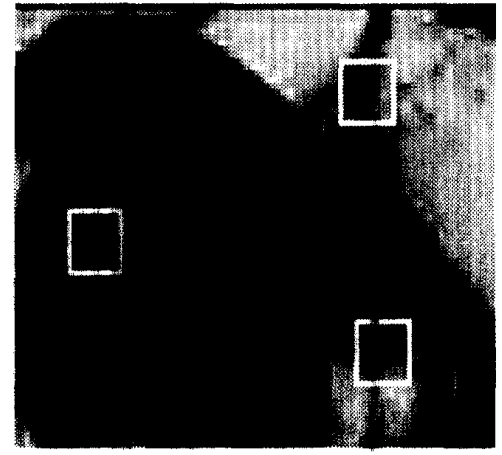

(c)

Fig. 4. Detail from the $256 \times 256$ peppers image shows a comparison (a) the original image with the image after compression at 0.5 b/pixel using (b) filter \#1 from Table I and (c) filter \#2 from Table I. Filter \#1 attains $30.83 \mathrm{~dB}$ PSNR versus $30.46 \mathrm{~dB}$ PSNR for filter \#3, yet filter \#3 achieves superior impulse preservation in the compressed image. The shadow impulses adjacent to the bright impulses in Fig. 4(c) are due to the odd symmetry of the highpass filter associated with filter $\# 3$.

eight $256 \times 256$ 8-b gray-scale test images were used, with the average PSNR calculated for a compression ratio of $16: 1$. These test images were pepper, lena, moon, bridge, couple, tank, diane, and geo. The first six are from the USC database, diane is a custom-scanned image that is lowpass in nature, and geo is an aerial photograph of a city provided by the U.S. Geological Survey.

We present in Table I, the results of our evaluation. For each filter bank, we give the the analysis $\left(H_{0}\right)$ and synthesis $\left(G_{0}\right)$ lowpass filter length, filter coefficients, and regularity. The impulse response peak to sidelobe ratio and step response second sidelobe strength are also given. We also give the average PSNR over eight test images using the compression algorithm mentioned above. The final column of the table gives the references (where applicable) to other publications where the filter is discussed, as well as comments. In addition to listing the best filter banks, we have also included two other filter banks that are of interest despite their poorer performance. Filter 8 is included to illustrate that regularity alone is not a sufficient criterion. For this filter, the synthesis filter regularity is quite high, but the compression performance is extremely poor. Fig. 3 shows that filter 8 introduces a significant amount of edge ringing into the compressed image, as well as periodic diamond shaped artifacts that may be due to aliasing in the 2-D highpass/highpass or "comer" detail signals. We also include (filter 7) the results for the Haar basis. The Haar basis shows that a filter with poor regularity can still achieve reasonably good compression if it has good impulse and step response properties.. Note that gain may be arbitrarily distributed between $H_{0}$ and $G_{0}$ as long as product of their dc gains is 2 . Filters 4 through 7 may be renormalized by a factor of $\sqrt{2}$ to have shifted integer coefficients, thus greatly simplifying hardware implementation.

We found that even length filters have significantly less shift variance than odd length filters, suggesting that they may be better for some applications. For example, it has been noted in [11] that even length filters reduce flicker noise in coded video images. Another desirable feature of even filters is their superior impulse response performance. Table I shows that the filters achieving the highest impulse response peak to sidelobe ratio are even length filters 7,5, and 3. For certain applications, it may be especially important to preserve impulses in the compressed image; for example, many geographic imaging systems introduce artificial reference dots and crosses into the image in order to indicate absolute location. Fig. 4 compares the impulse response preservation of images compressed with odd length filter number 1 (from Table I) and even length filter number 3 . Both have a total of 16 taps, and the odd filter slightly outperforms the even in PSNR and in perceived image quality. However, Fig. 4 shows that the even filter does a much better job of preserving location, shape, and intensity of impulses.

\section{CONCLUSIONS}

A multilevel analysis/synthesis wavelet filter bank can be evaluated by considering the reconstruction obtained from only the lowest level reference signal. This allows a description in terms of a linear, shift-variant system that can then be evaluated using criteria such as impulse and step response that are important in governing image quality. Although filter evaluation is made based only on the information preserved in the reference signal, the results remain valid when a full compression system employing quantization of both the reference and detail signals is used. Analysis of all reasonable length minimum order biorthogonal perfect reconstruction filters using these metrics identified filter banks that are well suited to image compression, including several that have not previously been published. While our interest was primarily in biorthogonal filters because of the greater analytical difficulty they present, the evaluation techniques we presented here could easily be applied to orthogonal filters or even to nonperfect reconstruction systems.

\section{REFERENCES}

[1] A. Grossmann and J. Morlet, "Decomposition of Hardy functions into square integrable wavelets of constant shape," SIAM J. Math. Anal., vol. 15, pp. 723-736, 1984.

[2] I. Daubechies, "Orthonormal bases of compactly supported wavelets," Commun. Pure Appl. Match., vol. XLI, pp. 909-996, 1988.

[3] S. Mallat, "A theory for multiresolution signal decomposition: The wavelet representation," IEEE Trans. Patt. Anal. Machine Intell., vol. 7, pp. 674-693, 1989. 
44] G. T. Strang, "Wayelets and dilation equations: A brief introduction," SIAM Rev., vol. 31, pp. 614-627, 1989.

[5] M. Antonini, M. Barlaud, P. Mathieu, and I. Daubechies, "Image coding using wavelet transform," IEEE Trans. Image Processing., vol. 1, pp. 205-220, 1992.

[6] P. Desarte, B. Macq, and D. Slock, "Signal-adapted multiresolution transform for image coding," IEEE Trans. Inform. Theory, vol. 38, pp. $897-903,1992$.

[7] O. Rioul, "Simple regularity criteria for subdivision schemes," SIAM J. Math Anal., vol. 23, pp. 1544-76, 1992.

[8] M. Vetterli and C. Herley, "Wavelets and filter banks: Theory and design," IEEE Trans. Signal Proc., vol. 40, pp. 2207-2232, 1992.

[9] A. H. Tewfik, D. Sinha, and P. Jorgensen, "On the optimal choice of a wavelet for signal representation," IEEE Trans. Inform. Theory, vol. 38, pp. 747-765, 1992.

[10] R. R. Coifman and M. V. Wickerhauser, "Entropy-based algorithms for best basis selection," IEEE Trans. Inform. Theory, vol. 38, pp. 713-718, 1992.

[11] T. Kronander, "New criteria for optimization of QMF banks to be used in an image coding system," in Proc. ISCAS '89, 1989, pp. 1354-1357.

[12] A. Cohen, I. Daubechies, and J. C. Feauveau, "Biorthogonal bases of compactly supported wavelets," AT\&T Bell Lab., Tech. Rep., TM 11217-900529-07, 1990.

[13] J. W. Woods and T. Naveen, "A filter-based bit allocation scheme for subband compression of HDTV," IEEE Trans. Image Processing, vol. 1, pp. 436-440, 1992

[14] E. P. Simoncelli, W. T. Freeman, E. H. Adelson, and D. J. Heeger, "Shiftable multiscale transforms," IEEE Trans. on Infor. Theory, vol. 38, pp. 587-607, 1992.

[15] T. Senoo and B. Girod, "Vector quantization for entropy coding of image subbands," IEEE Trans. Image Processing, vol. 1, pp. 526-533, 1992.

[16] J. Shapiro, "An embedded hierarchical image coder using zerotrees of wavelet coefficients," in Proc. IEEE Dafa Compression Conf. '93, Mar. 1993, pp. 214-223.

[17] D. Le Gall and A. Tabatabai, "Subband coding of digital images using symmetric short kernel filters and arithmetic coding techniques," in Proc. ICASSP '88, pp. 761-765, 1988.

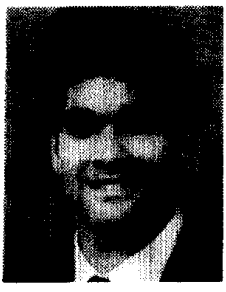

John D. Villasenor received the B.S. degree from the University of Virginia in 1985, the M.S. from Stanford University in 1986, and the Ph.D. from Stanford in 1989, all in Electrical Engineering. He has held postdoctoral appointments at Stanford and at CERFACS in Toulouse, France. From 1990 to 1992 Dr. Villasenor was with the Radar Science and Engineering section of the Jet Propulsion Laboratory in Pasadena, CA, where developed interferometric terrain mapping and classification techniques using synthetic aperture radar data. He is currently Assistant Professor of Electrical Engineering at the University of California, Los Angeles, and is conducting research on image and video compression with a focus on applications in wireless communications and in medicine.

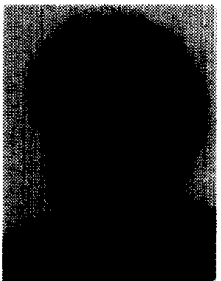

Benjamin Belzer received the B.A. degree in physics from the University of California at San Diego in 1982 and the M.S. degree in electrical engineering from the University of California at Los Angeles (UCLA) in 1993. He is currently working toward the Ph.D. degree with the UCLA Electrical Engineering Department.

Between 1981 and 1991, he worked as a software engineer for Beckman Instruments, Huges Aircraft, Northrop Corporation, and Source Scientific in Southern California and Develco, Inc. in Northern California. His current research interest are image compression, wavelet transforms and their applicationsto image processing, and wireless video communication systems.

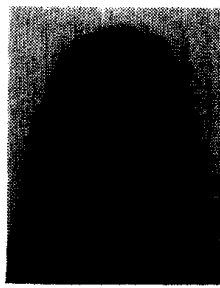

Judy Liao received the B.S. degree in electrical engineering and computer science from the University of California at Berkeley in 1989 and the M.S degree in electrical engineering from University of California at Los Angeles (UCLA) in 1993. She is currently working toward the $\mathrm{Ph} . \mathrm{D}$ degree with the UCLA Electrical Engineering Department. Her research interests are in the areas of algorithm development and simulation environments for lowbit-rate wirless image/video compression. 